\title{
Route Choice Model Considering Generalized Travel Cost Based on Game Theory
}

\author{
Feng Yu-qin, ${ }^{1}$ Leng Jun-qiang, ${ }^{2,3}$ Xie Zhong-Yu, ${ }^{1} \mathrm{Zhang} \mathrm{Gui-e,}^{3}$ and $\mathrm{He} \mathrm{Yi}^{3}$ \\ ${ }^{1}$ School of Automobile Engineering, Heilongjiang Institute of Technology, Harbin 150050, China \\ ${ }^{2}$ School of Management, Harbin Institute of Technology, Harbin 150001, China \\ ${ }^{3}$ School of Automobile Engineering, Harbin Institute of Technology, Weihai 264209, China \\ Correspondence should be addressed to Leng Jun-qiang; lengjunq@tom.com
}

Received 31 October 2012; Accepted 3 January 2013

Academic Editor: Matjaz Perc

Copyright (C) 2013 Feng Yu-qin et al. This is an open access article distributed under the Creative Commons Attribution License, which permits unrestricted use, distribution, and reproduction in any medium, provided the original work is properly cited.

\begin{abstract}
This paper aims at testing the influence of emission factors on travelers' behavior of route choice. The generalized travel cost is defined as the linear weighted sum of emission factors, travel time, and travel time reliability. The relational model of exhaust volume and traffic volume is established using the BPR (Bureau of Public Road) function to calculate the cost of travel regarding emission. The BPR function is used to measure the road segment travel time, while the reliability is used to quantify the cost of travel time fluctuation. At last, the route choice model considering the generalized travel cost is established based on the game theory. The calculating and analyzing of results under a miniature road network show that the weight coefficient of travel cost influences the travelers' behavior of route choice remarkably and the route choice model which takes emission into account can reduce the exhaust of road network effectively, approximately $11.4 \%$ in this case.
\end{abstract}

\section{Introduction}

How to reduce exhaust emission has become a very urgent subject as the number of automobile grows rapidly in China. At the moment, research on energy saving and emission reduction about the vehicle itself has been relatively mature, while few studies were conducted concerning this problem from the perspective of traffic management. The influence of emission factors on travelers' behavior of route choice will be studied in this paper from the aspect of traffic management, which can provide information about vehicle emission for traffic managers in order to decrease energy consumption and emission to some extent.

Travel time cost and fuel consumption are usually considered in travel cost estimation [1-3]. However, in general, former route choice models only took travel time cost into consideration. The researches on the relationship between route choice model and exhaust emission are few $[4,5]$. And there is only information about route length and travel time in the traffic guidance system and no other valuable information concerning emission. Travel cost was usually described using travel time which was presented by the BPR function.
There are some route choice models of travelers such as stochastic user equilibrium model and elastic demand equilibrium model [6]. As the prisoner's dilemma games and its extensions have been studied frequently [7-10], the paly game is also used in the traffic distribution [11]. However, their BPR functions do not well reflect different demands of travelers in choosing routes, because emission has gradually become a factor affecting route choice. And trip demand objectives are not single and are often mutually contradictory, for example, shorter travel time, higher reliability, and security $[12,13]$. Hence, generalized travel cost, which took emission into consideration, was studied in the paper based on the emission model for road segment. Then, the effect of route choice behavior and cost factors on route choice of travelers was discussed.

\section{Generalized Travel Cost Considering Emission}

Travelers are paying more attention to the emission problem in route choice besides the consideration of travel time and its fluctuation, because of the aggravation of the energy crisis 
and the pressing need of environmental protection. It is the ideal goal of travelers to reduce emission, decrease travel time, increase reliability, and so forth [14]. However, these goals often lead to contradiction and conflicts. Travelers are always seeking compromise in pursuing them. Therefore, this paper is based on the generalized travel cost of the above factors. The generalized travel cost is defined as the linear weighted sum of travelers' exhaust emission factor, travel time, and travel time reliability. So the generalized travel cost function may be represented as

$$
c_{a}=w_{1} l_{a} f_{a}(\cdot)+w_{2} T_{a}+w_{3} \gamma\left(T_{a}\right),
$$

where $c_{a}$ is the generalized travel cost in road segment $a ; f_{a}(\cdot)$ represents the emission factor on road segment $a,(\mathrm{~g} / \mathrm{km}) ; l_{a}$ is the length of road segment $a(\mathrm{~km}) ; T_{a}$ is the travel time of road segment $a(\mathrm{~s}) ; \gamma\left(T_{a}\right)$ is the travel time fluctuation of road segment $a ; w_{1}, w_{2}$, and $w_{3}$ are the weight of exhaust emission, travel time, and travel time reliability in the generalized travel cost, respectively. These three weight coefficients may reflect travelers' attitude towards hazards. The larger $w_{1}$ is, the smaller $w_{2}$ and $w_{3}$ will be, which means travelers pay more attention to emission, tending to consider emission as a route choice criterion. Each weight can be determined on the basis of SP (stated preference) survey.

The generalized travel cost of travelers can be represented as

$$
c_{k}^{w}=\sum_{a \in A} c_{a} \delta_{a k}^{w}
$$

where $c_{k}^{w}$ is the generalized travel cost of route $k$ in OD pair $w ; \delta_{a k}^{w}$ is a $0-1$ variable; if road segment $a$ lies in the route $k$ of the OD pair $w$, then $\delta_{a k}^{w}=1$; otherwise $\delta_{a k}^{w}=0$.

\subsection{Quantification of Travel Cost Index}

2.1.1. Travel Time. The BPR (Bureau of Public Roads) travel time function proposed by the United States Federal Highway Administration is the most representative achievement in the research of travel time model. The most significant influence of the model is on the field of traffic planning and it is most widely used in this field. It was initially used in the highway network planning, later in urban road network planning [15]. The mathematical expression is

$$
T_{a}=t_{a}\left[1+\beta\left(\frac{x_{a}}{C_{a}}\right)^{n}\right],
$$

where $T_{a}$ is the travel time of road segment $a,(\mathrm{~s}) ; t_{a}$ is the free flow travel time of road segment $a,(\mathrm{~s}) ; x_{a}$ is the traffic flow of road segment $a,(\mathrm{pcu} / \mathrm{h}) ; C_{a}$ is the traffic capacity of road segment $a,(\mathrm{pcu} / \mathrm{h}) ; \beta, n$ are the model parameters; the recommended values are $\beta=0.15, n=4$ in highway network application.

2.1.2. Travel Time Fluctuation. In 1991, Asakura and Kashiwadani [16] presented the travel time reliability concept to reflect travel time fluctuation. Travel time reliability is defined as the probability that a trip may be completed within a specified time under a certain LOS (level of service) demand, which is a measure of travel time stability index and describes the flexibility of road network in stochastic traffic conditions. In this paper, the travel time reliability is defined as

$$
R\left(T_{a}\right)=P\left(T_{a} \leq t_{a}+\Delta^{\prime}\right),
$$

where $\Delta^{\prime}$ is the acceptable threshold of buffer time; it usually takes $5 \%, 10 \%, 15 \%$, or $20 \%$ of the free travel time. For the simple reason that people have different tolerance degrees in various traffic congestions in different areas, $\Delta^{\prime}$ may be determined through the SP investigation; other parameters have the same meanings as previously mentioned. Substitute (3) into (4)

$$
R_{a}\left(T_{a}\right)=P\left[C_{a} \geq x_{a}\left(\frac{t_{a} \beta}{\Delta^{\prime}}\right)^{1 / n}\right] .
$$

Due to the capacity $C_{a}$ is a random variable, the distribution function $F_{C_{a}}(x)$ can be obtained by field observation; it may be written as

$$
\begin{gathered}
R_{a}\left(T_{a}\right)=1-F_{C_{a}}\left(x_{a}\left(\frac{t_{a} \beta}{\Delta^{\prime}}\right)^{1 / n}\right), \\
r\left(T_{a}\right)=1-R_{a}\left(T_{a}\right)=F_{C_{a}}\left(\left(\frac{t_{a} \beta}{\Delta^{\prime}}\right)^{1 / n} x_{a}\right) .
\end{gathered}
$$

In the formula, $r\left(T_{a}\right)$ is the probability of road segment $a$ not meeting the requirement of travel time reliability; it is deemed that $r\left(T_{a}\right)$ is the negative utility of travel time fluctuation.

2.1.3. Exhaust Emission Model. Average speed of vehicles is one of the most important factors that affect the exhaust emission. Margiotta [17] believed that emission factors were sensitive to speed and put forward the relational model between them. Wang et al. [18] calculated emission factors of vehicles under different average speeds using the modified MOBILE model based on the field data in Nanjing and then carried out a curve fitting taking emission factors as the dependent variable and the average speed of motor vehicle as the independent variable. The polynomial computational model for traffic exhaust emission can be written as follows:

$$
f_{a}=b_{0}+b_{1} v_{a}+b_{2} v_{a}^{2}+b_{3} v_{a}^{3},
$$

where $f_{a}$ represents emission factor of passenger car on road segment $a,(\mathrm{~g} / \mathrm{km}) ; v_{a}$ is the average speed of vehicles on road segment $a,(\mathrm{~km} / \mathrm{h}), 0 \leq v_{a} \leq 90 ; b_{0}, b_{1}, b_{2}$, and $b_{3}$ are regression coefficients.

Average speed of vehicles on a road segment can be calculated by travel time as follows, for the average speed of the vehicle equals road length divided by average travel time:

$$
v_{a}=\frac{l_{a}}{T_{a}}=\frac{l_{a}}{t_{a}\left[1+\beta\left(x_{a} / C_{a}\right)^{n}\right]},
$$

where $l_{a}$ is the length of road segment, $(\mathrm{km})$; other parameters are of the same meaning as mentioned previously. The volume 
of exhaust emission can be expressed as the function of the length of road segment, traffic volume, and its capacity.

Pluging (8) into (7), we can get the following model of the traffic volume and exhaust emission:

$$
\begin{aligned}
f_{a}= & b_{0}+b_{1}\left(\frac{l_{a}}{t_{a}\left[1+\beta\left(x_{a} / C_{a}\right)^{n}\right]}\right) \\
& +b_{2}\left(\frac{l_{a}}{t_{a}\left[1+\beta\left(x_{a} / C_{a}\right)^{n}\right]}\right)^{2} \\
& +b_{3}\left(\frac{l_{a}}{t_{a}\left[1+\beta\left(x_{a} / C_{a}\right)^{n}\right]}\right)^{3}
\end{aligned}
$$

2.2. Weight of Cost Index. Weight of different cost index may be determined on the basis of SP data, using the relative comparison method. First, make all indexes a square matrix. Second, compare every two indexes and score them, using $[0,1]$ scoring method. Third, sum up each index score and do the normalization. The weight of index $i$ may be expressed as

$$
w_{i}=\frac{\sum_{j=1}^{n} a_{i j}}{\sum_{i=1}^{n} \sum_{j=1}^{n} a_{i j}},
$$

where $a_{i j}$ is the importance of index $i$ relative to index $j$. Other parameters have the same meanings as mentioned previously.

2.3. Nondimensionalization of Indexes. To make different dimensional evaluation index comparable and additive, the method of mathematical transformation was used to eliminate the influence of index dimension in generalized travel cost. This paper uses linear proportion method to process nondimensionalization. The formula is

$$
y_{i}=\frac{x_{i}}{x_{i}^{\prime}}
$$

where $x_{i}$ is the sample value; $y_{i}$ is the dimensionless results of $x_{i}$; and $x_{i}^{\prime}$ is the minimum, maximum, or average value of the sample value.

\section{Game Theory Model}

The theory of games is a study about the interaction between rational decision makers. Each player has a number of strategies (feasible actions), which determines the outcome of the game and the payment to each player. In this game, a network user looks for a route to minimize the expected travel cost while an "evil entity" imposes road segment costs on the user so as to maximize the expected trip cost. This is assumed to be a two-player, noncooperative, and zero-sum game. The user guesses what road segment costs will be imposed and the evil entity guesses which route will be chosen. The mixed strategy that the Nash equilibrium proposed offers a useful measure for network reliability, since it yields the expected trip cost when the user is extremely pessimistic about the state of the network $[19,20]$.
We assume that situation $j$ implies performance degradation, failure, or attack on road segment $i$. The problem is to solve the following maximization and minimization model:

$$
\begin{aligned}
\min _{p_{i}} \max _{q_{j}} C & =\sum_{i j} p_{i} c_{i j} q_{j}=\sum_{i j} \sum_{k} \delta_{i k} h_{k} c_{i j} q_{j} \\
& =\sum_{k} h_{k} \sum_{j} g_{k j} q_{j}, \\
\text { St. } \sum_{j} q_{j} & =1 \\
q_{j} & \geq 0 \\
\sum_{k} \delta_{i k} h_{k} & =p_{i} \\
\sum_{k} h_{k} & =1 \\
h_{k} & \geq 0
\end{aligned}
$$

where $p_{i}$ is the probability that road segment $i$ is chosen; $q_{j}$ is the probability of situation $j ; c_{i j}$ is the travel cost of road segment $i$ under situation $j ; g_{i j}$ is the travel cost of path $k$ under situation $j ; h_{k}$ is the probability path $k$ is chosen; $\delta_{i k}$ equals 1 if road segment $i$ is on path $k, 0$ otherwise.

To facilitate the application of the route choice model, the method of successive average (MSA) scheme is used and it can be described as follows.

Step 1. Initialize $q_{j}$ for all situations $j$ and $n \leftarrow 1$.

Step 2. Set expected road segment costs to $\sum_{j} c_{i j} p_{j}$ for all road segments $i$.

Step 3. Build the least expected cost path; $x_{i} \leftarrow 1$ if road segment $i$ is on this path, and 0 otherwise.

Step 4. $p_{i} \leftarrow(1 / n) x_{i}+(1-1 / n) p_{i}$ for all road segments $i$.

Step 5. Find the $j$ which maximizes $\sum_{i} c_{i j} p_{i} ; y_{j} \leftarrow 1$; for all situations $k \neq j \quad y_{k} \leftarrow 0$.

Step 6. $q_{j} \leftarrow(1 / n) y_{i}+(1-1 / n) q_{j}$ for all situations $j$.

Step 7. $n \leftarrow n+1$ and return to Step 2 until a satisfactory convergence is achieved.

\section{Numerical Example}

A numerical example was chosen to verify the applicability of the model proposed in this paper. As shown in Figure 1, it includes 4 nodes, 5 road segments, and 1 OD pair $(1 \rightarrow$ 4). The value of OD pair is $2600 \mathrm{pcu} / \mathrm{h}$. In BPR function, parameters $\beta=0.15, n=4$, road segments length, free travel time, capacity and emission parameters, and so forth are shown in Table 1 . In the numerical example, $\Delta^{\prime}$ takes $15 \%$ of the free travel time $t_{a}$. Matlab 7.0 was used in the calculations.

The results show that the different attitudes of travelers towards travel cost have obvious effects on road network 
TABLE 1: Attribute data of road network unit.

\begin{tabular}{lccccc}
\hline Road segment & Road segment 1 & Road segment 2 & Road segment 3 & Road segment 4 & Road segment 5 \\
\hline Free travel time $t_{a} / \mathrm{s}$ & 76 & 70 & 49 & 76 & 49 \\
Capacity $C_{a} /(\mathrm{pcu} / \mathrm{h})$ & $N(1400,70)$ & $N(1100,90)$ & $N(1000,120)$ & $N(1400,70)$ & $N(1000,120)$ \\
Length $l / \mathrm{m}$ & 1600 & 1900 & 1200 & 1600 & 1200 \\
Parameters of emission model & & & & 168.351 & \\
$\quad b_{0}$ & 168.351 & 157.483 & 168.351 & -5.3423 & -5.3423 \\
$b_{1}$ & -5.3423 & -5.6240 & 0.0674 & 0.0674 & -0.0674 \\
$b_{2}$ & 0.0674 & 0.0609 & -0.0003 & -0.0003 & -0.0003 \\
$b_{3}$ & -0.0003 & -0.0003 & & & \\
\hline
\end{tabular}

TABLE 2: Volume under different weight coefficient (pcu/h).

\begin{tabular}{|c|c|c|c|c|c|c|c|}
\hline$w_{1}$ & $w_{2}$ & $w_{3}$ & Road segment 1 & Road segment 2 & Road segment 3 & Road segment 4 & Road segment 5 \\
\hline 0 & 1.0 & 0 & 662 & 1062 & 906 & 662 & 906 \\
\hline 0 & 0 & 1.0 & 843 & 962 & 795 & 843 & 795 \\
\hline 0.2 & 0.2 & 0.6 & 739 & 971 & 890 & 739 & 890 \\
\hline 0.2 & 0.4 & 0.4 & 641 & 1071 & 888 & 641 & 888 \\
\hline 0.2 & 0.6 & 0.2 & 767 & 1054 & 779 & 767 & 779 \\
\hline 0.2 & 0.8 & 0 & 705 & 994 & 901 & 705 & 901 \\
\hline 0.4 & 0.2 & 0.4 & 737 & 1083 & 781 & 737 & 781 \\
\hline 0.4 & 0.4 & 0.2 & 831 & 1031 & 738 & 831 & 738 \\
\hline 0.4 & 0.6 & 0 & 735 & 1004 & 861 & 735 & 861 \\
\hline 0.6 & 0.2 & 0.2 & 702 & 1044 & 854 & 702 & 854 \\
\hline 0.6 & 0.4 & 0 & 712 & 1030 & 857 & 712 & 857 \\
\hline 0.8 & 0.2 & 0 & 685 & 1076 & 840 & 685 & 840 \\
\hline 1.0 & 0 & 0 & 653 & 1043 & 904 & 653 & 904 \\
\hline
\end{tabular}

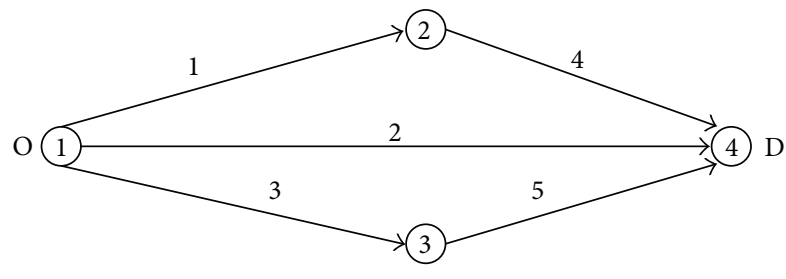

FIgURE 1: Test road network.

traffic assignment. From the results of Table 2, it can be found that when $w_{1}$ is gradually increasing until it approaches 1 , which means gradually increase the weight of emission factor in travel cost, the travelers tend to select emission as a criterion of route choice. Meanwhile, the travelers will select the low emission route as their priority selection. Because route $1-4$ is the longest, it is 1.68 times as long as route 2 and 1.33 times as long as route $3-5$, and it has the largest emission in terms of unit distance, so the emission cost of route $1-4$ is the highest. When $w_{1}$ is 1 , the distributed traffic capacity on route $1-4$ is the lowest; it is only $653 \mathrm{pcu} / \mathrm{h}$, but the traffic assignments on other routes are $1043 \mathrm{pcu} / \mathrm{h}$ and $904 \mathrm{pcu} / \mathrm{h}$. As a result, the emission of the whole local road network can be reduced by $11.4 \%$. When $w_{2}$ increases and approaches 1 , it indicates that travelers tend to select travel time as a criterion of route choice, and travelers tend to select the lower time-consuming route. Due to the fact that route 2 takes the shortest travel time, route 3-5 the next, and then route $1-4$, when $w_{2}$ is 1 , the distribution of traffic on route 2 is the highest, which is $1062 \mathrm{pcu} / \mathrm{h}$, route $3-5$ is $906 \mathrm{pcu} / \mathrm{h}$, and route $1-4$ is the lowest, which is only $662 \mathrm{pcu} / \mathrm{h}$. When $w_{3}$ gradually approaches 1 , it indicates that the travelers tend to treat reliability as a criterion. They will select the high-capacity and low-fluctuation route, and route 3-5 has the lowest capacity and highest fluctuation. Therefore, the assignment on route $3-5$ is the smallest, which is only $795 \mathrm{pcu} / \mathrm{h}$.

\section{Conclusions}

On the basis of the research carried out on emission model, road segment travel time, travel reliability, and generalized travel cost are defined and quantified. Meanwhile, the traffic distribution model that considers emission is proposed based on game theory. By means of the calculation examples, it is proved that travelers' different attitudes towards emission have obvious effects on route choice. The route choice model considering emission can distinctly reduce road network emission; in the example, it reduced by $11.4 \%$. With the popularized application of ITS, further researches about the effects of information and other factors on route choice behavior should be carried out. 


\section{Acknowledgments}

This research was supported by China Postdoctoral Science Foundation 2011M500676, National Education Ministry Humanities and Social Sciences Foundation 12YJCZH097, Heilongjiang Institute of Technology Doctoral Science Foundation 2011BJ05, Technology Research and Development Program of Shandong 2012G0020129, and Natural Science Foundation of Heilongjiang Province QC2011C060. The authors are also grateful to the anonymous referees for their helpful comments and constructive suggestions on an earlier version of the paper.

\section{References}

[1] S. Ghufran, S. Khowaja, and M. J. Ahsan, "Optimum multivariate stratified sampling designs with travel cost: a multiobjective integer nonlinear programming approach," Communications in Statistics, vol. 41, no. 5, pp. 598-610, 2012.

[2] G. Erdogan, M. Battarra, G. Laporte, and D. Vigo, "Metaheuristics for the traveling salesman problem with pickups, deliveries and handling costs," Computers and Operations Research, vol. 39, no. 5, pp. 1074-1086, 2012.

[3] V. Ovaskainen, M. Neuvonen, and E. Pouta, "Modelling recreation demand with respondent-reported driving cost and stated cost of travel time: a finnish case," Journal of Forest Economics, vol. 18, no. 4, pp. 303-317, 2012.

[4] S. Sugawara and D. A. Niemeier, "How much can vehicle emissions be reduced? Exploratory analysis of an upper boundary using an emissions-optimized trip assignment," Transportation Research Record, no. 1815, pp. 29-37, 2002.

[5] E. Ericsson, H. Larsson, and K. Brundell-Freij, "Optimizing route choice for lowest fuel consumption - potential effects of a new driver support tool," Transportation Research C, vol. 14, no. 6, pp. 369-383, 2006.

[6] C. M. Benedek and L. R. Rilett, "Equitable traffic assignment with environmental cost functions," Journal of Transportation Engineering, vol. 124, no. 1, pp. 16-22, 1998.

[7] Z. Wang and M. Perc, "Aspiring to the fittest and promotion of cooperation in the prisoner's dilemma game," Physical Review E, vol. 82, no. 2, Article ID 021115, 2010.

[8] M. Perc and Z. Wang, "Heterogeneous aspirations promote cooperation in the prisoner's dilemma game," PLOS ONE, vol. 5, no. 12, Article ID e15117, 2010.

[9] Z. Wang, A. Murks, W. B. Du, Z. H. Rong, and M. Perc, "Coveting thy neighbors fitness as a means to resolve social dilemmas," Journal of Theoretical Biology, vol. 277, no. 1, pp. 19-26, 2011.

[10] Z. Wang, S. Attila, and M. Perc, "Evolution of public cooperation on interdependent networks: the impact of biased utility functions," Europhysics Letters, vol. 97, no. 4, Article ID 48001, 2012.

[11] U. Naohiro and T. Eiichi, "A study of dispatcher's route choice model based on evolutionary game theory," Procedia, vol. 39, pp. 495-509, 2012.

[12] K. Ahn and H. Rakha, "The effects of route choice decisions on vehicle energy consumption and emissions," Transportation Research D, vol. 13, no. 3, pp. 151-167, 2008.

[13] G. H. Tzeng and C. H. Chen, "Multiobjective decision making for traffic assignment," IEEE Transactions on Engineering Management, vol. 40, no. 2, pp. 180-187, 1993.
[14] S. Zerguini, N. Khademi, and J. Shahi, "Variability of travel time, users' uncertainty, and trip information: new approach to costbenefit analysis," Transportation Research Record, no. 2254, pp. 160-169, 2011.

[15] L. Engelson, "Properties of expected travel cost function with uncertain travel time," Transportation Research Record, no. 2254, pp. 151-159, 2011.

[16] Y. Asakura and M. Kashiwadani, "Road network reliability caused by daily fluctuation of traffic flow," in Proceedings of the 19th PTRC Summer Annual Meeting, pp. 73-84, Brighton, UK, September 1991.

[17] R. A. Margiotta, Improved Vehicle Speed Estimation Procedures for Air Quality and Planning Application, The University of Tennessee, Knoxville, Tenn, USA, 1996.

[18] W. Wang, Q. J. Xiang, and T. Z. Li, Urban Transport System Energy Consumption and Environmental Impact Analysis, Science Press, Beijing, China, 2003.

[19] V. Bioglio, R. Gaeta, M. Grangetto, M. Sereno, and S. Spoto, "A game theory framework for ISP streaming traffic management," Performance Evaluation, vol. 68, pp. 1162-1174, 2011.

[20] L. J. Sun and Z. Y. Gao, "An equilibrium model for urban transit assignment based on game theory," European Journal of Operational Research, vol. 181, no. 1, pp. 305-314, 2007. 


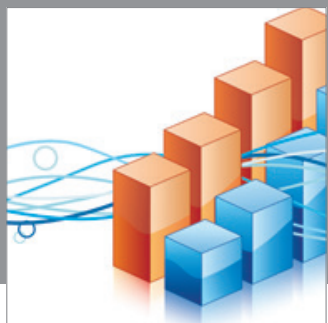

Advances in

Operations Research

mansans

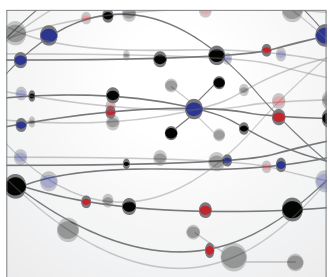

The Scientific World Journal
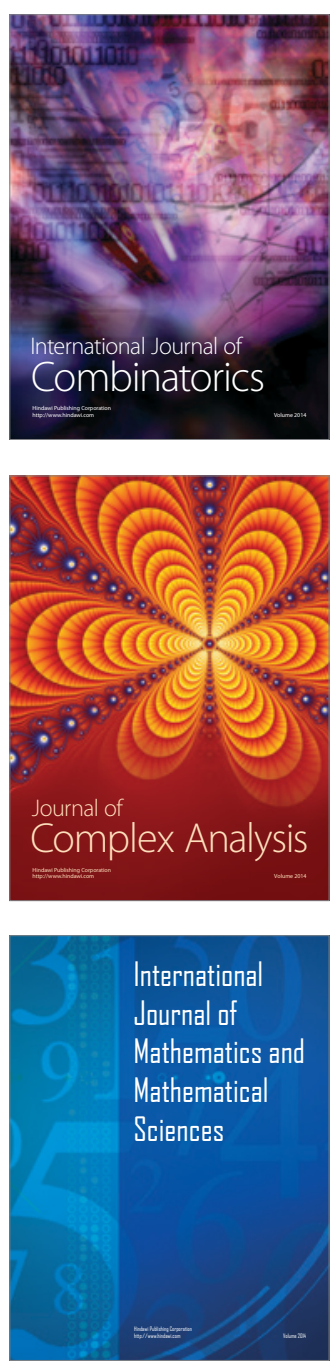
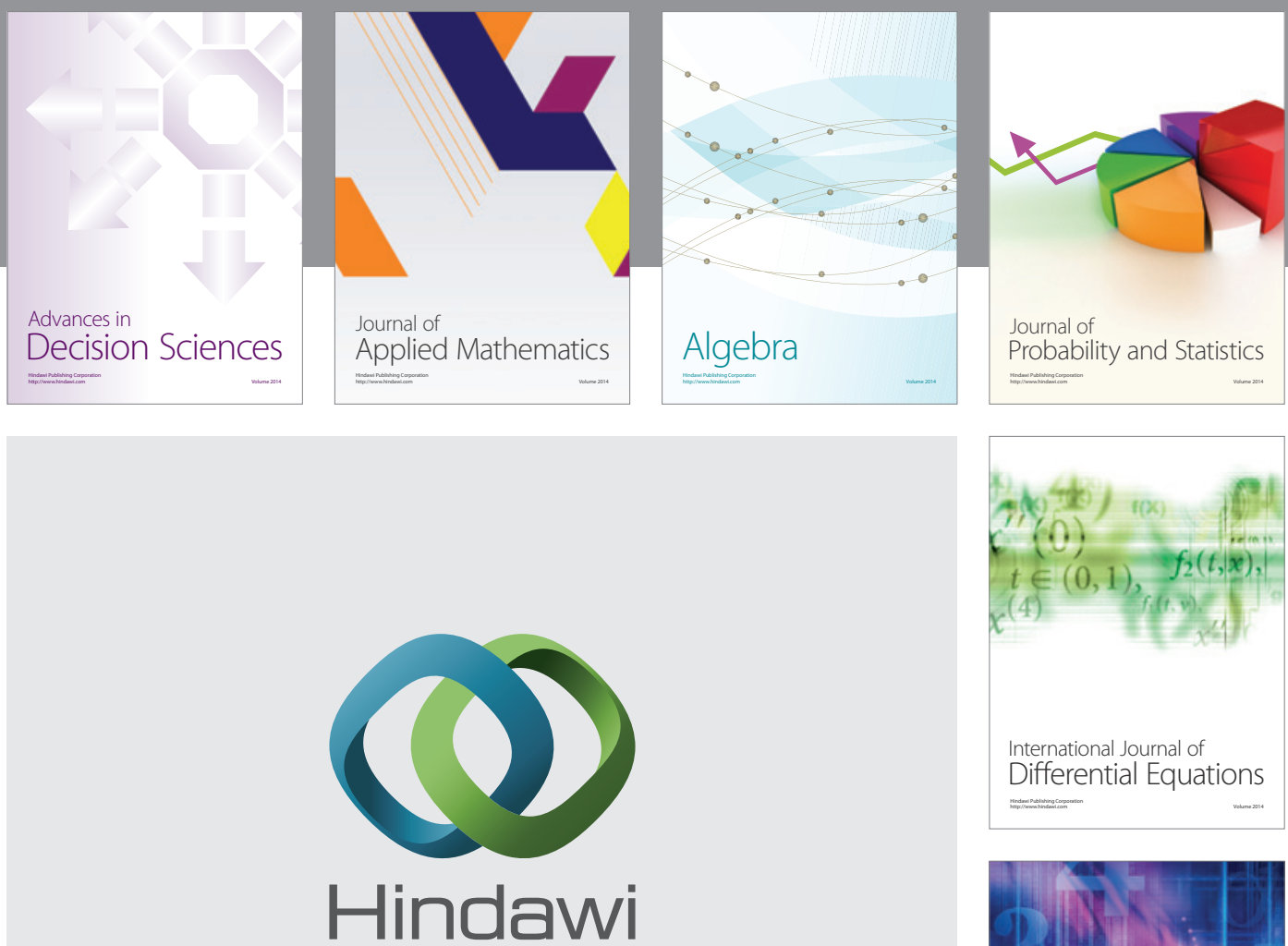

Submit your manuscripts at http://www.hindawi.com
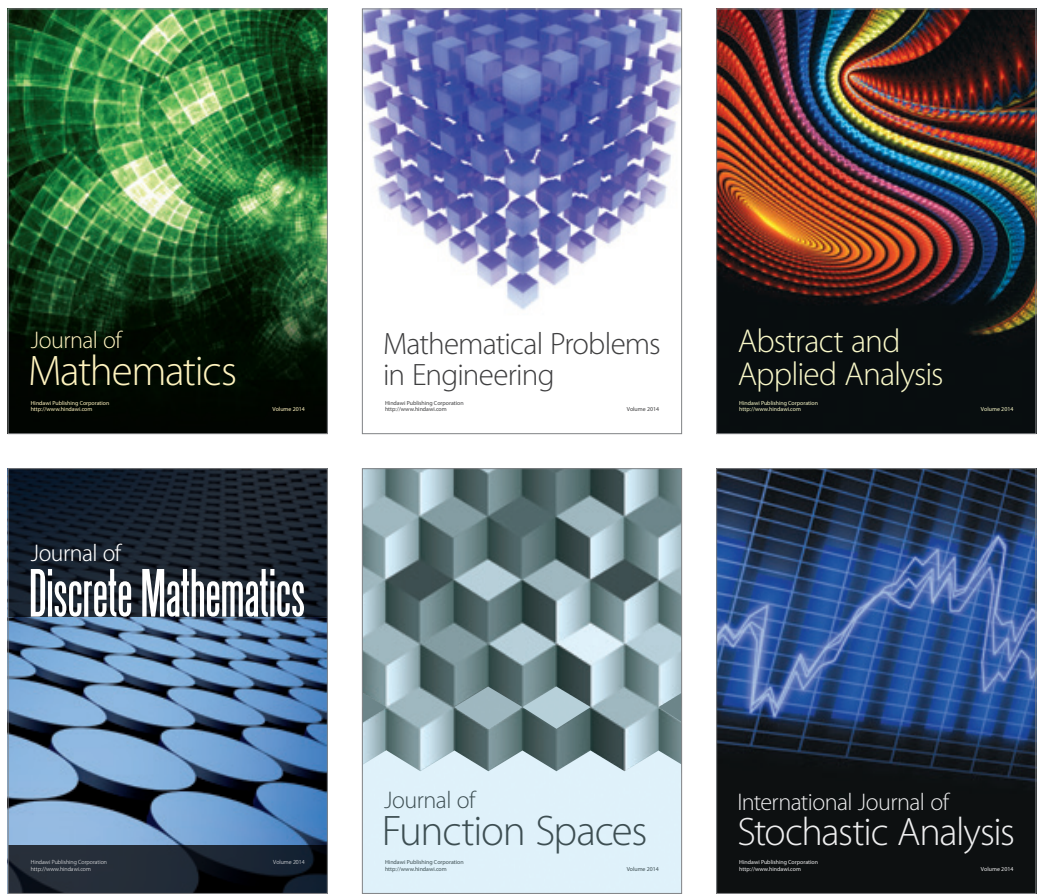

Journal of

Function Spaces

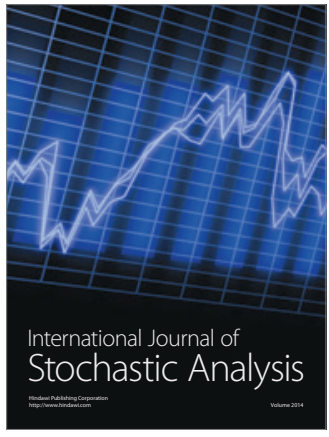

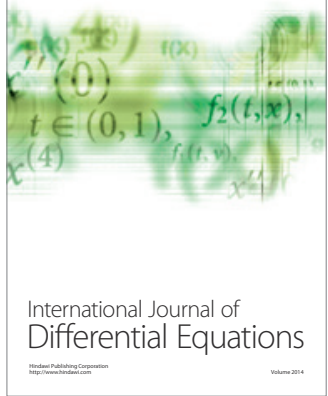
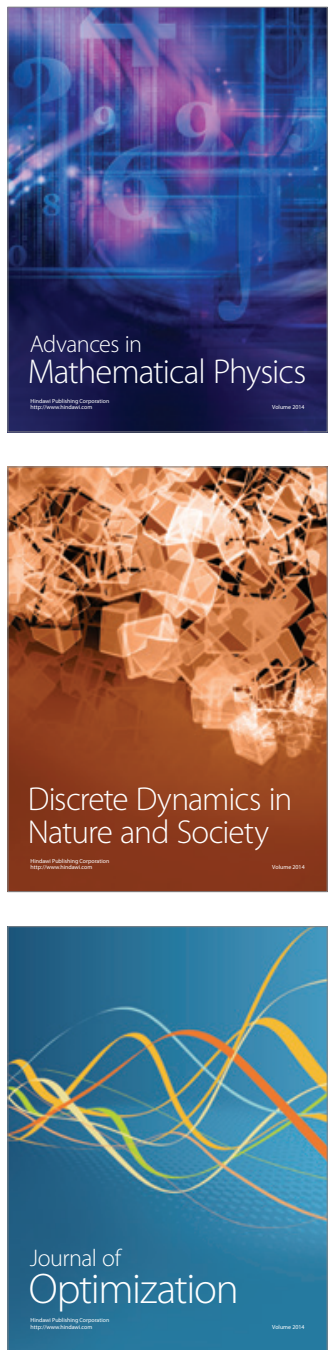